\title{
MGP and EGP Rings
}

\section{Raida D. Mahammod}

\author{
Shahla M. Khalil
}

raida.1961@uomosul.edu.iq

moayadshahla@gmail.com

College of Computer Sciences and Mathematics

University of Mosul, Iraq

\section{Received on: 12/3/2008}

Accepted on: 12/6/2008

\section{ABSTRACT}

The purpose of this paper is to study the rings in which every maximal (essential) right ideal is a left GP- ideal. Such rings will be called right MGP- rings (EGP- rings). We give the basic properties of such rings and their connection with strongly $\pi$-regular rings, fully left idempotent rings, and $S$ - weakly regular rings.

Keywords: right MGP- rings,right EGP- rings, strongly $\pi$ - regular rings, fully left idempotent rings, $S$ - weakly regular rings.

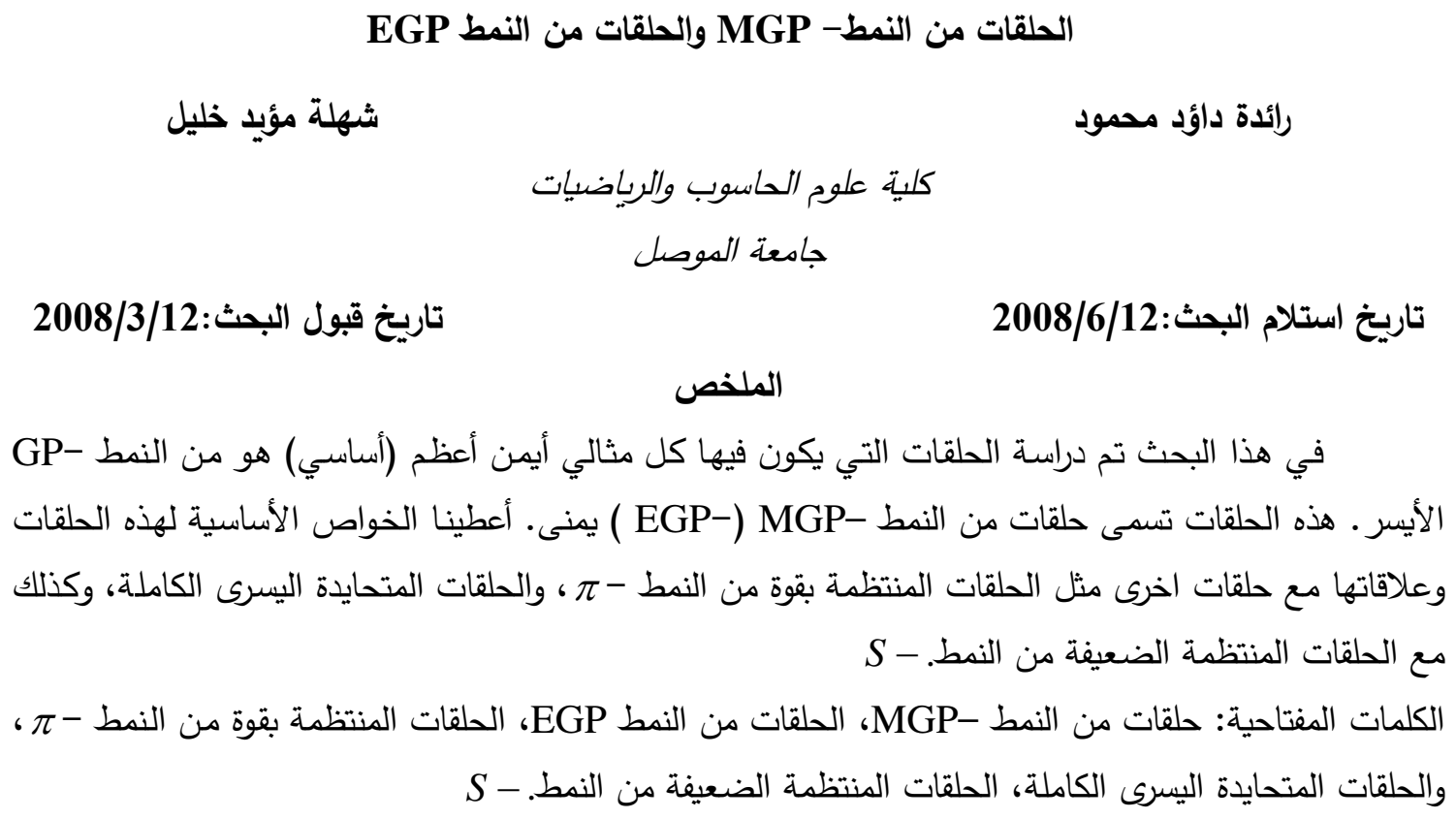

\section{Introduction:}

In this paper, all rings are assumed to be associative ring with identity. An ideal $I$ of a ring $\mathrm{R}$ is said to be right (left) pure ideal if for every $a \in I$ there exists $b \in I$ such that $a=a b(a=b a)$. This concept was introduced by Fieldhouse [3],[4].

\section{Recall that:}

1 - $\mathrm{R}$ is called reduced if $\mathrm{R}$ has no non-zero nilpotent element.

2- According to Cohn [2], a ring $\mathrm{R}$ is called reversible if $a b=0$ implies $b a=0$, for $a, b \in R$. It is easy to see that $\mathrm{R}$ is reversible if and only if right (left) annihilator of $a$ in $\mathrm{R}$ is two sided ideal [2].

3- A ring $\mathrm{R}$ is said to be a right (left) Kasch ring if every maximal right (left) ideal is a right (left) annihilator [8]. 
4- According to [5], a ring $\mathrm{R}$ is called strongly $\pi$-regular if for every $x \in R$, there exists a positive integer $n$ such that $x^{n} R=x^{2 n} R$.

5- A ring $\mathrm{R}$ is called right (left) quasi-duo if every maximal right (left) ideal of $\mathrm{R}$ is two sided ideal [1].

6- A ring $\mathrm{R}$ is said to be ERT if every essential right ideal is two-sided [1].

\section{2- Maximal Generalized Pure Rings (MGP- rings)}

In this section, some basic properties of MGP- rings are given. Also the relations between such rings and strongly $\pi$ - regular, weakly $\pi$-regular rings are given.

Following [10], an ideal $I$ of a ring $\mathrm{R}$ is said to be right (left) GP- ideal if for every $a \in I$, there exists $b \in I$ and a positive integer $n$ such that $a^{n}=a^{n} b\left(a^{n}=b a^{n}\right)$.

Definition 2.1.[10]: A ring $\mathrm{R}$ is called a right (left) MGP- rings if and only if every maximal right (left) ideal is left (right) GP-ideal.

Theorem 2.2: Let $\mathrm{R}$ be a ring with $l\left(a^{n}\right) \subseteq r(a)$, for any $a \in R$ and a positive integer $n$. If $\mathrm{R}$ is a right MGP- ring, then the Jacobson radical of $\mathrm{R}$ is zero $(J(R)=(0))$.

Proof: Let $0 \neq a \in J(R)$. If $a R+r(a) \neq R$, then there exists a maximal right ideal $M$ containing $a R+r(a)$. Since $\mathrm{R}$ is right MGP- ring, then $M$ is left GP-ideal, so there exists $b \in M$ and a positive integer $n$ such that $a^{n}=b a^{n},(1-b) a^{n}=0$ hence $(1-b) \in l\left(a^{n}\right) \subseteq r(a) \subseteq M$. So $1 \in M$, a contradiction. Therefore $a R+r(a)=R$, and so $a r+d=1$ for some $r \in R$ and $d \in r(a)$, this implies that $a=a^{2} r$. Since $a \in J(R)$, then there exists an invertible element $v$ in R such that $(1-a r) v=1$, so $\left(a-a^{2} r\right) v=a$ yields $a=0$. This proves that $J(R)=0$. \#

Theorem 2.3: Let $M$ be a maximal right ideal of $R$. Then $R$ is MGP- ring if and only if for every $a \in M, M+l\left(a^{n}\right)=R$ for some positive integer $n$.

Proof: Let $M$ be a maximal right ideal of $R$ and $a \in M$, since $R$ is MGP- ring, then $M$ is a left GP- ideal so there exists $b \in M$ such that $a^{n}=b a^{n}$ for some positive integer $n$. This implies that $(1-b) \in l\left(a^{n}\right)$. Therefore $R=M+l\left(a^{n}\right)$.

Conversely, assume that $M+l\left(a^{n}\right)=R$ for every $a \in M$ and a positive integer $n$, then $t+s=1$ for some $t \in M$ and $s \in l\left(a^{n}\right)$. So $t a^{n}+s a^{n}=a^{n}$ and this implies $a^{n}=t a^{n}$. Whence $R$ is MGP- ring. \#

Lemma 2.4.[6]: Every strongly $\pi$-regular is $\pi$-regular ring.

Recall that, $\mathrm{R}$ is said to be W.R.D (weakly right duo), if for each $a \in R$ there exists a positive integer $n$ such that $a^{n} R=R a^{n} R$.

Theorem 2.5.[7]: Let R be W.R.D ring. Then the following are equivalent:

$1-\mathrm{R}$ is a $\pi$-regular.

2- Every ideal of $\mathrm{R}$ is a left GP-ideal.

Theorem 2.6: If $\mathrm{R}$ is a right Kasch ring and reversible, then the following statements are equivalent:

1 - $\mathrm{R}$ is a strongly $\pi$ - regular ring.

2- $\mathrm{R}$ is a right MGP- ring. 
Proof: $(1) \Rightarrow(2)$ :

Assume that $\mathrm{R}$ is strongly $\pi$ - regular ring then by Lemma 2.4 and Theorem 2.5, $\mathrm{R}$ is a right MGP- ring.

$(2) \Rightarrow(1)$ :

Assume that $\mathrm{R}$ is a right MGP- ring. Let $M$ be any maximal right ideal of $\mathrm{R}$. Since $\mathrm{R}$ is a right Kasch ring, then $M=r\left(a^{n}\right)$ for some $a \in R$ and a positive integer $n$. For any $x \in M$, we have $a^{n} x=0$, and so $a^{n} R x=0$. This implies that $R x \subseteq r\left(a^{n}\right)=M$, which proves that $M$ is a two sided ideal of $\mathrm{R}$. We claim that $b^{n} R+r\left(b^{n}\right)=R$. If not, there is a maximal right ideal $N$ of $\mathrm{R}$ such that $b^{n} R+r\left(b^{n}\right) \subseteq N$. Since R is MGP- ring, then $N$ is a left GP-ideal and $b^{n} \in N$. Then there exists $y \in N$ such that $b^{n}=y b^{n}$. Hence $(1-y) b^{n}=0$ and so $(1-y) \in l\left(b^{n}\right)=r\left(b^{n}\right) \subseteq N$ (because $\mathrm{R}$ is reversible). Thus $1 \in N$, a contradiction. Therefore $b^{n} R+r\left(b^{n}\right)=R$. In particular $b^{n} u+v=1$ for some $u \in R$ and $v \in r\left(b^{n}\right)$, so $b^{n}=b^{2 n} u$. Thus $\mathrm{R}$ is strongly $\pi$-regular. \#

Theorem 2.7: Let $\mathrm{R}$ be a right Kasch ring and reversible. If $\mathrm{R}$ is a right MGP- ring. Then for each completely prime ideal $P$ of $\mathrm{R}, P=\underset{x \notin P}{\cup} r(x)$.

Proof: By Theorem 2.6, $\mathrm{R}$ is a strongly $\pi$-regular ring. Let $Q=\underset{x \notin P}{\cup} r(x)$ and show that $Q=P$. If $x \in Q$, then $x \in r(y)$ for some $y \notin P$, thus $y x=0 \in P$ and hence $x \in P$. Therefore $Q \subseteq P$.

On the other hand, by the Lemma $2.4, P$ is $\pi$ - regular ideal, thus for each $x \in P$, there exists $u \in P$ and a positive integer $n$ such that $x^{n}=x^{n} u x^{n}$, which implies that $x^{n}\left(1-u x^{n}\right)=0 \in P$. Then $x^{n} \in l\left(1-u x^{n}\right)=r\left(1-u x^{n}\right)$ (because $\mathrm{R}$ is reversible ring). Since $\left(1-u x^{n}\right) \notin P$ for otherwise $1 \in P$, which is impossible. Thus $x^{n} \in Q$, so that $P \subseteq Q$, whence $Q=P$.\#

Following [9], a ring $\mathrm{R}$ is called right (left) weakly $\pi$ - regular if, for every $x \in R$ there exists a positive integer $n$ such that $x^{n} \in x^{n} R x^{n} R \quad\left(x^{n} \in R x^{n} R x^{n}\right)$. R is weakly $\pi$ regular if it is both right and left weakly $\pi$-regular.

The next result gives us a sufficient condition for MGP- ring to be weakly $\pi$ regular ring.

Theorem 2.8: Let $\mathrm{R}$ be a semi prime ring with each non-zero right ideal contains a nonzero two sided ideal. If $\mathrm{R}$ is an MGP-ring, then it is weakly $\pi$-regular ring.

Proof: Assume that $0 \neq a \in R$ such that $a^{2}=0$, then by assumption there is a non-zero two sided ideal of $\mathrm{R}$ with $I \subseteq a R$. We claim that $l(a) \cap I \neq(0)$, for if $I a=0$ then $I \subseteq l(a)$ and we are done. If $I a \neq 0$, then $I a \subseteq I \cap l(a) \neq(0)$ Now, $(I \cap l(a))^{2} \subseteq l(a) I \subseteq l(a) a R=(0)$. Since $\mathrm{R}$ is semi prime ring, then $I \cap l(a)=(0)$ which is a contradiction, consequently $\mathrm{R}$ is reduced. We show that $R x^{n} R+r\left(x^{n}\right)=R$ for any $x \in R$ and a positive integer $n$. Suppose that there exists $y \in R$ such that $R y^{n} R+r\left(y^{n}\right) \neq R$. Then there exists a maximal right ideal $M$ of $\mathrm{R}$ containing 
$R y^{n} R+r\left(y^{n}\right)$. Since $\mathrm{R}$ is a right MGP- ring, then $M$ is a left GP-ideal. So there exists $a \in M$ and a positive integer $m$ such that $\left(y^{n}\right)^{m}=a\left(y^{n}\right)^{m}$ implies $(1-a) \in l\left(\left(y^{n}\right)^{m}\right)=r\left(\left(y^{n}\right)^{m}\right)=r\left(y^{n}\right) \subseteq M$. Hence $(1-a) \in M$ and so $1 \in M$ implies that $M=R$ which is a contradiction. Therefore $R x^{n} R+r\left(x^{n}\right)=R$ for any $x \in R$. In particular $c x^{n} d+u=1$ for some $u \in r\left(x^{n}\right)$ and $c, d \in R$, hence $x^{n}=x^{n} c x^{n} d$. So $\mathrm{R}$ is a right weakly $\pi$-regular ring. Since $\mathrm{R}$ is reduced it is also can be easily verified that $\mathrm{R}$ is weakly $\pi$-regular ring. \#

Theorem 2.9.[6]:Let $\mathrm{R}$ be duo ring. Then $\mathrm{R}$ is $\pi$-regular if and only if $\mathrm{R}$ is strongly $\pi$ - regular.

Lemma 2.10.[13]:If $\mathrm{R}$ is left or right quasi- duo and $J(R)=(0)$. Then $\mathrm{R}$ is reduced ring.

Proposition 2.11.[6]: Let R be W.R.D. Then the following statement are equivalent:

1 - $\mathrm{R}$ is a weakly $\pi$-regular ring.

$2-\mathrm{R}$ is a strongly $\pi$ - regular ring.

The following theorem extends Theorem 2.6.

Proposition 2.12: Let $\mathrm{R}$ be a right duo ring. Then the following statements are equivalent:

$1-\mathrm{R}$ is a strongly $\pi$-regular ring.

$2-\mathrm{R}$ is a $\pi$-regular ring.

$3-\mathrm{R}$ is a right weakly $\pi$ - regular ring.

4- $\mathrm{R}$ is a right MGP- ring and $J(R)$ is nil.

Proof: $(1) \Rightarrow(2)$ : Follows from Theorem 2.9.

(2) $\Rightarrow(3)$ : Obvious.

(3) $\Rightarrow$ (4) : Follows from Proposition 2.11 and Theorem 2.5. Thus every maximal right ideal is GP-ideal. Now, we show that $J(R)$ is nil.

Let $r \in J(R)$. Then $r^{n} R=r^{n} R r^{n} R$ for some positive integer $n$, and so $r^{n}(1-s)=0$ for some $s \in R r^{n} R$. But since $r \in J(R),(1-s)$ is invertible and thus we have $r^{n}=0$, showing that $J(R)$ is nil.

(4) $\Rightarrow(1)$ : Assume (4), then from Theorems 2.5 and 2.9, R is strongly $\pi$-regular. \#

Lemma 2.13.[10]: Let $\mathrm{R}$ be a reduced ring. Then every GP- ideal is pure ideal.

By the Proposition 2.12, Lemmas 2.10 and 2.13, we have the following theorem.

Theorem 2.14: Let $\mathrm{R}$ be a right quasi-duo and $J(R)=0$. Then the following statements are equivalent:

$1-\mathrm{R}$ is a strongly $\pi$ - regular ring.

$2-\mathrm{R}$ is a $\pi$-regular ring.

3 - $\mathrm{R}$ is a right (left) weakly $\pi$ - regular ring.

4- $\mathrm{R}$ is a weakly $\pi$-regular ring.

5- $\mathrm{R}$ is a right MGP- ring.

6- Every maximal right ideal is a left pure.

\section{3- EGP- rings}


In this section, we introduce a new essential GP- ideals which are called EGP- rings. We give some of their basic properties, as well as a connection between EGP- rings and $S$ - weakly regular rings, strongly regular rings.

Definition 3.1: A ring $\mathrm{R}$ is said to be right EGP- rings, if every essential right ideal of $\mathrm{R}$ is a left GP- ideals.

Recall that [11], a ring $\mathrm{R}$ is a right (left) $S$ - weakly regular if for each $a \in R$, $a \in a R a^{2} R \quad\left(a \in R a^{2} R a\right)$. A ring $\mathrm{R}$ is called $S$ - weakly regular ring if it is both right and left $S$ - weakly regular ring.

We start this section by recalling the following propositions:

Proposition 3.2.[12]: A ring $\mathrm{R}$ is $S$ - weakly regular ring if and only if $\mathrm{R}$ is a reduced weakly regular ring.

Proposition 3.3.[10]: Let $\mathrm{R}$ be a duo ring. Then $\mathrm{R}$ is regular if and only if every ideal $I$ of $\mathrm{R}$ is left pure.

Now, the following result is given:

Theorem 3.4: Let $\mathrm{R}$ be a ring with $a R=R a$. Then $\mathrm{R}$ is a reduced, right EGP- ring if and only if $\mathrm{R}$ is an $S$ - weakly regular ring.

Proof: Assume that $\mathrm{R}$ is a reduced right EGP- ring. Let $a \in R$ and $I=R a^{2} R+r(a)$. We claim that $I$ is an essential right ideal of R. Suppose this is not true, then there exists a non-zero ideal $J$ of R such that $I \cap J=(0)$. Then $\left(R a^{n} R\right) J \subseteq I J \subseteq I \cap J=(0)$. Since $a^{2} R \subseteq R a^{2} R$, then $a^{2} R \cap J=(0)$. But $\left(a^{2} R\right) J \subseteq a^{2} R \cap J=(0)$ implies $J=(0)$, a contradiction; hence $I$ is an essential right ideal. Since R is right EGP- ring, then $I$ is a left GP- ideal, for every $a \in I$ there exists $b \in I$ and a positive integer $n$ such that $a^{n}=b a^{n}$. Since $b \in I$ and $I=R a^{2} R+r(a)$, then $b=c a^{2} d+h$ for some $c, d \in R$ and $h \in r(a)$ hence $a^{n}=b a^{n}=c a^{2} d a^{n}+h a^{n}$. Since $\mathrm{R}$ is reduced then $r(a)=l(a)=l\left(a^{n}\right)$. So $a^{n}=c a^{2} d a^{n}+0 \quad$ implies that $\quad\left(1-c a^{2} d\right) a^{n}=0 \quad$ and $\left(1-c a^{2} d\right) \in l\left(a^{n}\right)=r\left(a^{n}\right)=r(a)$ hence $a=a c a^{2} d$. Therefore $\mathrm{R}$ is an $S-$ weakly regular ring.

Conversely, assume that $\mathrm{R}$ is an $S$ - weakly regular ring. Then by Proposition $3.2, \mathrm{R}$ is reduced weakly regular ring. Let $a \in R$ and $a R=a R a R=a R R a=a R a$ since $(a R=R a)$. So $\mathrm{R}$ is regular and by Proposition 3.3, $\mathrm{R}$ is EGP- ring. \#

Lemma 3.5.[9]: Let $\mathrm{R}$ be a weakly regular ring. Then $J(R)=(0)$.

However, we have the following proposition:

Proposition 3.6: Let $\mathrm{R}$ be a right duo ring. Then the following statements are equivalent:

$1-\mathrm{R}$ is a strongly regular ring.

$2-\mathrm{R}$ is a regular ring.

$3-\mathrm{R}$ is a right weakly regular ring.

$4-\mathrm{R}$ is an $S$ - weakly regular ring.

5- $\mathrm{R}$ is a right EGP- ring and $l\left(a^{n}\right) \subseteq r(a)$ for every $a \in R$ and a positive integer $n$.

Proof: $(1) \Rightarrow(2) \Rightarrow(3)$ : They are obvious. 
(3) $\Rightarrow(4)$ : Assume (3), then by Lemmas 3.5, 2.10 and Proposition 3.2, R is an $S$ - weakly regular ring.

(4) $\Rightarrow(5)$ : It follows from Theorem 3.4.

(5) $\Rightarrow(1)$ : Assume that $\mathrm{R}$ is right EGP- ring. For any $a \in T$, let $T=a R+r(a)$ be a right ideal, by a similar method of the proof used in Theorem 3.4, $T$ is an essential ideal. Since R is a right EGP-ring, then $T$ is a left GP- ideal. For every $a \in T$, there exists $b \in T$ and a positive integer $n$ such that $a^{n}=b a^{n}$, which implies $(1-b) \in l\left(a^{n}\right) \subseteq r(a) \subseteq T$, so $1 \in T$ and $T=R$. Therefore $a R+r(a)=R$.

In particular $a r+d=1$ for some $r \in R$ and $d \in r(a)$. Then $a=a^{2} r$. Thus $\mathrm{R}$ is strongly regular ring. \#

Definition 3.7.[8]: A ring $\mathrm{R}$ is called fully right (left) idempotent if every right (left) ideal of $\mathrm{R}$ is idempotent.

Theorem 3.8: If $\mathrm{R}$ is ERT, then the following condition are equivalent:

$1-\mathrm{R}$ is a fully left idempotent ring.

$2-\mathrm{R}$ is a right EGP- ring.

Proof: (1) $\Rightarrow(2)$ : Assume (1), and let $E$ be an essential right ideal of $\mathrm{R}$ then it is an ideal of $\mathrm{R}$ (since $\mathrm{R}$ is ERT). Since $\mathrm{R}$ is a fully left idempotent ring, then for any $x \in E$, $R x=(R x)^{2}$ which implies that $x=a x$ for some $a \in R x R \subseteq E$. Therefore $x \in E x$ for each $x \in E$. So $E$ is left pure implies $E$ is a left GP- ideal. Thus R is EGP- ring.

(2) $\Rightarrow(1)$ : Assume (2), for any $a \in R$, set $L=R a^{n} R+l\left(R a^{n} R\right)$, and let $K$ be a complement right ideal of $\mathrm{R}$ such that $L \oplus K$ is an essential right ideal of $\mathrm{R}$. Now, $K R a^{n} R \subseteq K \cap R a^{n} R \subseteq K \cap L=(0) \quad$ implies that $K \subseteq l\left(R a^{n} R\right)$. Whence $K \subseteq K \cap L=(0)$. This shows that $L$ is an essential right ideal of $\mathrm{R}$ which is an ideal of $\mathrm{R}$, by hypothesis $\mathrm{R}$ is a right EGP- ring. Therefore $L$ is left GP- ideal and $a \in L$ implies that $a^{n}=d a^{n}$ for some $d \in L$ and a positive integer $n$. If $d=u+v, u \in R a^{n} R$ and $\quad v \in l\left(R a^{n} R\right)$, then $a^{n}=u a^{n}+v a^{n}=u a^{n} \in R a^{n} R a^{n} \quad$ which implies that $a^{n} \in\left(R a^{n}\right)^{2}$; whence $R a^{n}=\left(R a^{n}\right)^{2}$. Therefore $\mathrm{R}$ is a fully left idempotent ring. \# 
[1] Brown, S.H. (1973), "Rings over which every simple module is rationally complete", Canad. J. Math. 25, pp. 693-701.

[2] Cohn, P.M. (1999), "Reversible rings", Bull. London Math. Soc. 31, pp.641648.

[3] Fieldhouse, D.J. (1969), "Pure theories", Math. Ann. 184, pp. 1-18.

[4] Fieldhouse, D.J. (1970), "Pure simple and indecomposable rings", Con. Math. Ball. 13, pp. 71-78.

[5] Hirano, Y. (1978), "Some studies on strongly $\pi$-regular rings", Math. J. Okayama Univ. 20, pp. 141-144

[6] Mahammad, M.R. (2000), "On $\pi$-regular rings", Ph.D. Thesis, University of Mosul.

[7] Mahmood, R.D. and Khalil, S.M. (2008), "ON GP-IDEALS", No. 34. Under publication.

[8] Ming, R.Y.C. (1986), "On semi prime and reduced ring", Riv. Math. Univ. Parama(4) 12, pp.167-175.

[9] Ramamurthi, V.S. (1973), "Weakly regular rings", Canda Math. Bull. 16, pp. 317-321.

[10] Shuker, N.H. and Mahmood, R.D. (2000), "On Generalization of pure ideal", J. Edu. And Sci, Vol. (43), pp. 86-90.

[11] Vasantha Kandasamy, W.B. and Gupta, V. (1993), " $S$-weakly regular group rings", Avch. Math. (BRNO) Tomus 29, pp.39-41.

[12] Younis, A.M. (2003), "On $S$-weakly regular ring", M. Sc. Thesis, Mosul University.

[13] Yu, H. P. (1995), "On quasi-duo", Glasgow Math. J. 37, pp. 21-31. 